\title{
Efeito do creep feeding sobre o desempenho de bezerros e a eficiência reprodutiva de primíparas Nelore, em pastejo
}

[Effect of creep feeding on average daily gain and weaning weight of calves and on reproductive efficiency of primiparous Nelore cows under grazing]

\author{
E. Nogueira ${ }^{1,4}$, M.G. Morais $^{2}$, V.J. Andrade ${ }^{3}$, E.D.S. Rocha ${ }^{4}$, A.S. Silva ${ }^{5}$; A.T. Brito ${ }^{5}$ \\ ${ }^{1}$ Aluno de pós-graduação - FCAV - UNESP \\ Via de Acesso Prof. Paulo Donato Castellane, $\mathrm{s} / \mathrm{n}$ \\ 14884-900 - Jaboticabal, SP \\ ${ }^{2}$ Departamento de Produção Animal - UFMS - Campo Grande, MS \\ ${ }^{3}$ Escola de Veterinária -UFMG - Belo Horizonte, MG \\ ${ }^{4} \mathrm{UCDB}, \mathrm{MS}$, Campo Grande, MS \\ ${ }^{5}$ Gêneses Reprodução Animal
}

\begin{abstract}
RESUMO
Avaliou-se o efeito da suplementação de bezerros em sistema de creep feeding, em pastagens de Brachiaria brizantha, durante o período de amamentação, sobre o ganho médio diário (GMD), peso à desmama (PD) e taxa de gestação, em delineamento inteiramente ao acaso, utilizando 102 vacas Nelore (primíparas de baixa condição corporal ao início da estação de monta) e seus bezerros, divididos em dois grupos: T1 $(\mathrm{n}=52)$, não tratado e T2 $(\mathrm{n}=50)$, tratado com suplemento à base de $20 \%$ de $\mathrm{PB}$ e $75 \%$ de NDT. O consumo médio diário estimado no período foi $0,61 \mathrm{~kg}$ de suplemento/bezerro/dia. Observaramse diferenças entre T1 e T2 quanto ao $\mathrm{PD}(\mathrm{P}<0,01)$ e GMD $(\mathrm{P}<0,05)$, com valores, respectivamente, de $155,10 \pm 2,72$ e $163,80 \pm 2,53 \mathrm{~kg}$; e $0,59 \pm 0,015$ e $0,64 \pm 0,014 \mathrm{~kg}$. A taxa de gestação não foi influenciada pelos tratamentos $(\mathrm{P}>0,05)$. A suplementação em creep feeding pode aumentar o GMD e o PD de bezerros Nelore, sem alterar a taxa de gestação de primíparas que iniciam a estação de monta com baixa condição corporal.
\end{abstract}

Palavras-chave: bovino, Nelore, creep feeding, ganho de peso, pré-desmama, taxa de gestação

\begin{abstract}
The effect of creep feeding on average daily gain (ADG) and weaning weight $(W W)$ of calves and pregnancy rate of dams was evaluated in Nelore cattle on Brachiaria Brizantha pasture. In a complete randomized design, calves were divided into two treatments: T1, the control group and T2, in which calves were provided a supplemental diet containing 20\% CP and 75\% TDN from 92 days after birth until weaning. The 102 primiparous cows were in low body condition at beginning of breeding season. Average daily consumption of the creep ration was $0.61 \mathrm{~kg}$ per calf. $W W$ averaged $155.10 \pm 2.72 \mathrm{~kg}$ and $163.80 \pm 2.53$ and for $T 1$ and $T 2$ calves, respectively $(P<0.01)$; while ADG averaged $0.59 \pm 0.015 \mathrm{~kg}$ and $0.64 \pm 0.014$ respectively $(P<0.05)$. Pregnancy rate did not differ between treatment groups $(P>0.05)$. Thus, creep feeding can improve $W W$ and preweaning $A D G$ of Nelore calves but may not affect pregnancy rate of primiparous cows in low body condition at the start of the mating.
\end{abstract}

Keywords: creep feeding, preweaning growth, pregnancy rate, Nelore, primiparous cows

Recebido em 5 de outubro de 2004.

Aceito em 10 de agosto de 2005

E-mail: eriklis@yahoo.com 


\section{INTRODUÇÃO}

A necessidade de produzir novilhos precoces e/ou superprecoces e de aumentar a taxa de desfrute dos rebanhos aumenta a importância de se produzir animais com elevado peso à desmama. O ganho diário dos bezerros no início da lactação constitui um indicativo da quantidade de leite produzido pela vaca (Boggs et al., 1980). A relação entre ganho de peso médio diário (GMD) do bezerro e a produção de leite da mãe diminui depois de 16 semanas (Leal e Freitas, 1982). Segundo esses autores, na raça Charolesa essa relação deixa de ser significativa a partir do terceiro mês de idade. Acredita-se que, a partir de três a quatro meses de idade, parte dos nutrientes necessários ao bezerro de corte venha de outras fontes que não o leite materno.

Fordyce et al. (1996), ao trabalharem com animais Brahman e mestiços, encontraram diferença de $10,8 \%$ no GMD a favor dos bezerros suplementados com $16 \%$ de proteína bruta e $66 \%$ de NDT. O consumo diário de suplemento foi de $0,40 \mathrm{~kg}$ por animal. Em outro experimento, os mesmos autores não observaram diferenças significativas no GMD e no peso à desmama (PD) de bezerros suplementados por 42 dias e com consumo de $0,10 \mathrm{~kg} / \mathrm{dia} / \mathrm{animal}$, indicando que o GMD e o PD dos animais suplementados em creep feeding estão relacionados com a quantidade de suplemento ingerido.

Martin et al. (1981) utilizaram 831 bezerros Angus para avaliar os efeitos do creep feeding sobre o peso à desmama, ganho de peso pósdesmama e sobre a fertilidade das vacas. Houve diferença a favor dos bezerros suplementados quanto ao peso às idades de 120, 210 e 365 dias. Pacola et al. (1989) estudaram os efeitos da suplementação em creep feeding sobre os pesos de 495 bezerros Nelore aos 120 e 210 dias, sobre a fertilidade e o peso das vacas à parição e à desmama. A suplementação dos bezerros que se iniciou aos dois meses de idade e prosseguiu por 122 dias resultou em efeito favorável sobre o peso à desmama. As vacas cujos bezerros foram suplementados em creep feeding apresentaram tendência de maior ganho de peso até a desmama e melhor eficiência reprodutiva.

Em primíparas, reservas energéticas inadequadas à parição podem ser mais agravantes para a retomada da atividade cíclica ovariana do que em vacas adultas, pois demanda adicional para a continuidade do crescimento, somada ao estresse da primeira lactação, pode exacerbar esse problema (Spitzer et al., 1995). A variação do escore corporal e do peso do animal durante a estação de monta influencia a retomada da atividade ovariana e a taxa de concepção (Swayer et al., 1991). Outro fator de importância para a retomada dessa atividade é a ação inibitória da mamada do bezerro sobre os reguladores da liberação do Gnrh.

Segundo Fordyce et al. (1996), a suplementação de bezerros em creep feeding até a desmama pode diminuir a ingestão de leite e, pela redução do estímulo da mamada, provocar retorno mais cedo da atividade ovariana pós-parto. Jolly et al. (1994) verificaram que a intensidade de mamada foi maior quando vacas meio-sangue zebu foram alimentadas com dietas de pior qualidade, em conseqüência da menor produção leiteira. Os valores encontrados foram: 3,6 e 4,8kg para a produção diária de leite; 13,7 e 9,5 minutos para cada episódio de mamada e 127 e 92 minutos por dia para tempo de amamentação para os grupos com baixo e alto nível nutricional, respectivamente. Os autores sugeriram que o aumento da intensidade da mamada aumentou o feedback negativo sobre a secreção de gonadotrofinas, retardando o reinício da atividade ovariana pós-parto.

Lishman et al. (1984b) demonstram que a suplementação em creep feeding por períodos variáveis de 2,5 a 4,5 meses pode melhorar as taxas de concepção das matrizes. Prichard et al. (1989), ao trabalharem com vacas multíparas, cujos bezerros foram suplementados ou não em creep feeding, não verificaram diferenças entre os grupos na taxa de prenhez, encontrando maior peso à desmama nos animais suplementados. Lishman et al. (1984a) e Pacola et al. (1989) constataram pequeno aumento, não significativo, na fertilidade de vacas primíparas como resultado da suplementação de seus bezerros em creep feeding.

Os objetivos deste trabalho foram avaliar os efeitos da suplementação de bezerros Nelore, em fase de amamentação, filhos de vacas primíparas, sobre o GMD e o PD, bem como o peso e as taxas de prenhez de suas mães ao final da estação de monta. 


\section{MATERIAL E MÉTODOS}

O experimento foi realizado na Fazenda Cristo, de propriedade da Agropecuária Nove de Ouro, no município de Miranda - MS. A região apresenta clima subúmido tipo AW de Köeppen, pluviosidade média anual de $1400 \mathrm{~mm}$ concentrada no verão. A precipitação pluviométrica durante o experimento mostrou uma situação atípica, com valor baixo para o mês de janeiro (Tab. 1).

Tabela 1. Precipitação pluviométrica mensal (mm) durante o período experimental - Miranda - MS

\begin{tabular}{lc}
\hline Mês & $\mathrm{mm}$ \\
\hline Dezembro/1999 & 176 \\
Janeiro/2000 & 10 \\
Fevereiro/2000 & 124 \\
Março/2000 & 268 \\
Abril/2000 & 56 \\
Maio/2000 & 39 \\
Total & 673 \\
\hline
\end{tabular}

De um lote de 200 novilhas foram selecionadas, por exames ginecológicos e datas de parto, 102 primíparas Nelore de três anos de idade e 92 dias pós-parto e seus respectivos bezerros. Os animais foram distribuídos em dois tratamentos: T1controle (52 vacas primíparas sem suplementação dos bezerros), T2-creep feeding (50 vacas primíparas cujos bezerros receberam concentrado contendo $20 \%$ de PB e $75 \%$ de NDT). O suplemento foi fornecido aos bezerros na proporção de $1 \%$ do peso vivo e em função do consumo do dia anterior, em espaço cercado, aonde suas mães não tinham acesso, e próximo ao cocho de sal mineral. A suplementação iniciou-se com a estação de monta (84 dias) e terminou com a desmama, totalizando 135 dias. O consumo foi avaliado diariamente com a pesagem da quantidade fornecida e das sobras restantes nos cochos. Utilizou-se a monta natural com dois touros/lote, avaliados andrologicamente, na relação touro vaca de 1:25. A identificação dos animais em cio foi feita em duas observações diárias, às 7 e às 17 horas, com duração média de 90 minutos em cada observação. O diagnóstico de gestação foi realizado via palpação transretal, cerca de 50 dias após a retirada dos touros.
As vacas foram pesadas e avaliadas quanto ao escore corporal utilizando-se uma escala de 1-9, segundo Wiltbank (1983), no início do experimento, para a formação dos lotes, e a cada 30 dias, totalizando seis avaliações.

Utilizou-se um delineamento inteiramente ao acaso. As informações de PD, GMD e os pesos das vacas ao final da estação de monta foram submetidos à análise de variância, utilizando-se o procedimento GLM do SAS (User's... 1996). Como somente quatro nascimentos ocorreram no mês de agosto, para a análise dos dados, estes foram agrupados com os de setembro. Os dados da taxa de gestação foram avaliados pelo teste de qui-quadrado.

Para PD e GMD, utilizou-se o seguinte modelo matemático:

$\mathrm{Y}_{\mathrm{ijkl}}=\mu+\mathrm{T}_{\mathrm{i}}+\mathrm{S}_{\mathrm{j}}+\mathrm{N}_{\mathrm{k}}+(\mathrm{TS})_{\mathrm{ij}}+\beta\left(\mathrm{Pi}_{\mathrm{l}}-\mathrm{Pi}\right)+\mathrm{e}_{\mathrm{ijkl}}$, em que:

$\mathrm{Y}_{\mathrm{ijkl}}=$ variáveis dependentes (PD e GMD);

$\mu=$ efeito médio;

$\mathrm{T}_{\mathrm{i}}=$ efeito do i-ésimo tratamento ( $\mathrm{i}=\mathrm{T} 1$ e T2);

$\mathrm{S}_{\mathrm{j}}=$ efeito do j-ésimo sexo ( $\mathrm{j}=$ machos e fêmeas);

$\mathrm{N}_{\mathrm{k}}=$ efeito do k-ésimo mês de nascimento ( $\mathrm{k}=$ setembro, outubro e novembro);

$\beta=$ coeficiente de regressão linear da característica, incluída no modelo como covariável;

$\mathrm{Pi}_{1}=$ peso do bezerro ao início da suplementação; $\mathrm{Pi}=$ média de peso dos bezerros ao início da suplementação;

$\mathrm{e}_{\mathrm{ijkl}}=$ erro associado à $\mathrm{ijkl}$ observação

\section{RESULTADOS E DISCUSSÃO}

O consumo médio de concentrado por bezerro suplementado no creep feeding foi de $0,61 \mathrm{~kg}$, perfazendo um consumo total de $82,35 \mathrm{~kg}$ durante o período experimental. $\mathrm{O}$ consumo médio diário mensal foi de $0,11,0,25,0,56,1,01$ e 1,20kg para os meses de janeiro, fevereiro, março, abril e maio, respectivamente, aumentando com $\mathrm{o}$ aumento da idade dos bezerros, embora inferior ao fornecido ( $1 \%$ do peso vivo), aspecto já observado por Siqueira et al. (2001). 
Pacola et al. (1989) observaram aumento do consumo de suplemento com o aumento da idade dos bezerros. O consumo médio diário (CMD) foi de $0,328 \mathrm{~kg} /$ animal $/$ dia durante todo o período (122 dias), variando de 0,066 durante o primeiro mês de suplementação, quando a idade média dos bezerros era de três meses, para $0,747 \mathrm{~kg}$ no último mês (idade de sete meses). O consumo médio de suplemento de $1 \mathrm{~kg} / \mathrm{dia} /$ bezerro só foi alcançado após o terceiro mês de suplementação, quando os animais do T2 apresentavam $135 \mathrm{~kg}$ de peso vivo.

Em dois experimentos na Austrália, com animais em condições extensivas, Fordyce et al. (1996) encontraram CMD de 0,1 e $0,4 \mathrm{~kg} /$ dia durante 42 dias, utilizando um concentrado que continha $16 \%$ de PB e $65 \%$ de NDT. Segundo esses autores, o baixo consumo observado foi atribuído principalmente ao comportamento de pastejo do rebanho. Os autores registraram os principais horários de pastejo no início da manhã e no final da tarde. Os bezerros, ao acompanharem suas mães, estavam em aleitamento e consumiam pasto, o que pode ter diminuído o interesse pelo suplemento oferecido no cocho.

O GMD dos bezerros durante todo o período de suplementação foi de $0,64 \pm 0,088 \mathrm{~kg}$. A análise de variância das variáveis independentes incluídas no modelo é apresentada na Tab. 2. Observou-se efeito de tratamento $(\mathrm{P}<0,05)$ e sexo do bezerro $(\mathrm{P}<0,01)$ sobre o GMD. O creep feeding proporcionou aumento no GMD total $(\mathrm{P}<0,05)$, contudo não foram observadas diferenças na avaliação para cada mês do período experimental. $\mathrm{O}$ maior GMD observado no período de fevereiro a março (Tab. 2) faz supor que houve ganho compensatório, como resultado da maior disponibilidade quali-quantitativa de alimento, conforme observado por Nogueira et al. (2003a,b), provocado pelo aumento da precipitação nesse mês (Tab. 1) e, provavelmente, pela melhoria na produção de leite das matrizes.

Registrou-se diferença $(\mathrm{P}<0,01)$ entre sexos no T2 para GMD total, isto é, os machos desse grupo ganharam $0,09 \mathrm{~kg}$ a mais que as fêmeas. Também ganharam 0,08 e $0,11 \mathrm{~kg}$ a mais que os machos e as fêmeas do $\mathrm{T} 1$, respectivamente, indicando melhor aproveitamento da suplementação pelos bezerros machos (Tab. 3).

Lishman et al. (1984b) observaram aumento de 0,10kg no GMD de bezerros suplementados em creep feeding em relação aos animais do grupocontrole. Em relação ao GMD e de acordo com o sexo, os autores encontraram superioridade de apenas $0,005 \mathrm{~kg}$ dos machos suplementados em relação às fêmeas suplementadas, inferior aos resultados obtidos neste trabalho.

Tabela 2. Ganho médio diário de acordo com os tratamentos e mês de avaliação

\begin{tabular}{llccccc}
\hline Tratamento & $\mathrm{N}$ & Jan* & Fev-mar & Mar-abr & Abr-mai & Total \\
\hline T1 & 52 & $0,41 \pm 0,03 \mathrm{a}$ & $0,83 \pm 0,04 \mathrm{a}$ & $0,59 \pm 0,05 \mathrm{a}$ & $0,59 \pm 0,04 \mathrm{a}$ & $0,59 \pm 0,01 \mathrm{~b}$ \\
$\mathrm{~T} 2$ & 50 & $0,44 \pm 0,03 \mathrm{a}$ & $0,79 \pm 0,03 \mathrm{a}$ & $0,69 \pm 0,04 \mathrm{a}$ & $0,66 \pm 0,03 \mathrm{a}$ & $0,64 \pm 0,02 \mathrm{a}$ \\
\hline
\end{tabular}

Valores seguidos de letras distintas na coluna diferem entre si; $\mathrm{SNK}(\mathrm{P}<0,01)$.

T1: controle; T2: bezerros suplementados em creep feeding.

* Primeira pesagem realizada em 08/01/00, e as demais a cada 30 dias.

Tabela 3. Ganho médio diário (GMD) e peso à desmama (PD) dos bezerros por sexo, de acordo com o tratamento

\begin{tabular}{lllll}
\hline Tratamento & Sexo & N & GMD $(\mathrm{kg})$ & PD $(\mathrm{kg})$ \\
\hline T1 & Macho & 24 & $0,61 \pm 0,02 \mathrm{~b}$ & $155,37 \pm 3,60 \mathrm{~b}$ \\
& Fêmea & 28 & $0,58 \pm 0,02 \mathrm{~b}$ & $154,84 \pm 3,46 \mathrm{~b}$ \\
T2 & Macho & 26 & $0,69 \pm 0,02 \mathrm{a}$ & $170,29 \pm 3,23 \mathrm{a}$ \\
& Fêmea & 24 & $0,60 \pm 0,02 \mathrm{~b}$ & $157,31 \pm 3,55 \mathrm{~b}$
\end{tabular}

Valores seguidos de letras distintas na coluna diferem entre si $(\mathrm{P}<0,01)$.

T1: controle; T2: bezerros suplementados em creep feeding. 
Quando foi avaliado o efeito do tratamento sobre o peso à desmama em função do sexo dos bezerros, observou-se superioridade para T2 $(\mathrm{P}<0,01)$ de $12,98 \mathrm{~kg}$ para os machos em relação às fêmeas do mesmo grupo, e de 14,92 e 15,45kg em relação a machos e fêmeas do $\mathrm{T} 1$, respectivamente (Tab. 3). Não houve diferença no PD entre as fêmeas do T2 e machos e fêmeas do $\mathrm{T} 1(\mathrm{P}>0,05)$. Estes resultados discordam de vários autores (Pacola et al., 1977, 1989), que verificaram que o tratamento em creep feeding foi eficiente em aumentar o peso à desmama de bezerros machos e fêmeas. Os resultados deste trabalho concordam parcialmente com Martin et al. (1981), que encontraram superioridade maior dos machos suplementados (200 vs 180) em relação às fêmeas suplementadas (176 vs 166), demonstrando que animais com maior potencial de crescimento, beneficiados pelo dimorfismo sexual, obtêm melhores respostas pela suplementação em creep feeding.

$\mathrm{O}$ tratamento apresentou efeito $(\mathrm{P}<0,01)$ no peso dos bezerros apenas à desmama (idade média de $226,34 \pm 19,18$ dias) quando a diferença entre os grupos foi de $8,73 \mathrm{~kg}$ em favor do T2. Não houve diferenças nas pesagens anteriores (Tab. 4). Pacola et al. (1989) afirmaram que a suplementação de bezerros começa a apresentar resultado mais expressivo a partir do quarto mês de idade, quando a suplementação se inicia aos dois meses, o que não foi observado neste experimento.

O resultado para peso à desmama é inferior aos relatados por Pacola et al. (1989), que encontraram, para bezerros azebuados (Guzerá), aumento de $13,0 \mathrm{~kg}$ no peso à desmama, a favor dos animais suplementados, e por Pacola et al. (1977), que verificaram aumento de $26,8 \mathrm{~kg}$ para os bezerros suplementados. Vale ressaltar que, nesse último experimento, o consumo de suplemento foi de $1,157 \mathrm{~kg} /$ bezerro/dia e que a suplementação foi realizada durante o período da seca, quando a resposta à suplementação é maior devido às piores condições de pastagens.

Registrou-se diferença $(\mathrm{P}<0,05)$ no peso das vacas ao final da estação de monta, com superioridade de $7,66 \mathrm{~kg}$ para as vacas que tiveram seus bezerros suplementados (Tab. 5). Pacola et al. (1989) e Fordyce et al. (1996) também relataram aumento do peso das matrizes cujos bezerros receberam suplementação em creep feeding.

Tabela 4. Peso de bezerros por mês $(\mathrm{kg})$, de acordo com o tratamento

\begin{tabular}{lccccc}
\hline Tratamento & Janeiro & Fevereiro & Março & Abril & Maio \\
\hline T1 & $71,53 \pm 1,98 \mathrm{a}$ & $89,70 \pm 0,91 \mathrm{a}$ & $113,84 \pm 1,50 \mathrm{a}$ & $132,26 \pm 1,88 \mathrm{a}$ & $155,10 \pm 2,73 \mathrm{a}$ \\
T2 & $74,95 \pm 1,88 \mathrm{a}$ & $90,74 \pm 0,86 \mathrm{a}$ & $113,63 \pm 1,38 \mathrm{a}$ & $135,02 \pm 1,75 \mathrm{a}$ & $163,83 \pm 2,53 \mathrm{~b}$ \\
\hline
\end{tabular}

Valores seguidos de letras distintas na coluna diferem entre si $(\mathrm{P}<0,05)$.

T1: controle; T2: bezerros suplementados em creep feeding.

Tabela 5. Pesos (kg) e escore corporal (EC) das vacas de acordo com os tratamentos

\begin{tabular}{|c|c|c|c|c|c|}
\hline Tratamento & & $\begin{array}{c}8 / 01 / 00 \\
\text { (início EM) }\end{array}$ & $9 / 02 / 00$ & $9 / 03 / 00$ & $\begin{array}{l}8 / 04 / 00 \\
\text { Fim EM }\end{array}$ \\
\hline T1 & Peso & $282,78 \pm 4,36 a$ & 259,96 & 287,38 & $291,57 \pm 2,54 b$ \\
\hline $\mathrm{T} 2$ & Peso & $283,07 \pm 4,10 \mathrm{a}$ & 256,7 & 292,76 & $299,23 \pm 2,41 a$ \\
\hline T1 & $\mathrm{EC}$ & 4,23 & 3,26 & 4,44 & 4,65 \\
\hline $\mathrm{T} 2$ & LC & 4,05 & 3,46 & 4,55 & 4,86 \\
\hline
\end{tabular}

EM= estação de monta. T1: controle; T2: bezerros suplementados em creep feeding.

Valores seguidos de letras distintas na coluna diferem entre si $(\mathrm{P}<0,05)$.

As taxas de prenhez $(28,8 \%$ para T1 e $42,0 \%$ para T2) não foram influenciadas pelos tratamentos.

Pacola et al. (1989) encontraram tendência de aumento na fertilidade das matrizes (aumento de
5,5\% na taxa de prenhez em favor do grupo suplementado $) \quad(\mathrm{P}>0,05)$ como resultado da suplementação dos bezerros em creep feeding. Lishman et al. (1984 a), em dois experimentos na África do Sul, com vacas multíparas Africander, cujos bezerros receberam $0,9 \mathrm{~kg} / \mathrm{dia}$ de 
suplemento do quarto ao sétimo mês de idade, ou com primíparas Africander e Hereford, cujos bezerros foram suplementados nos 4,5 últimos meses de lactação, registraram que a suplementação em creep feeding, embora tenha aumentando o peso à desmama, não apresentou efeito sobre o peso das matrizes em ambos os estudos. Com relação à taxa de gestação, no estudo com multíparas, não foi registrado efeito do creep feeding. No estudo com primíparas, a taxa de gestação foi aumentada de 32 para $60 \%$, em função da suplementação de seus bezerros, demonstrando que essa é a categoria animal que melhor responde à suplementação em creep feeding, como também observado por Pacola et al. (1989).

O aumento de peso das matrizes ao final da estação de monta bem como o aumento da fertilidade podem ser atribuídos ao efeito da suplementação dos bezerros, promovendo redução do estímulo da mamada e, conseqüentemente, dos efeitos inibitórios sobre a secreção de gonadotrofinas pelos centros hipotalâmicos e hipofisários. Com isso, o retorno da atividade ovariana pós-parto foi mais rápido, associado à menor perda de peso das vacas, conforme citado por Fordyce et al. (1996).

As baixas taxas de prenhez podem ser atribuídas ao peso e a escore corporal das primíparas, no início da estação de monta, e também à perda de peso das fêmeas no mês de fevereiro, em conseqüência da baixa precipitação pluviométrica observada no mês de janeiro. Segundo Richards et al. (1989), a ingestão inadequada de nutrientes provoca a perda de peso e de condição corporal, com inibição da atividade lútea e cessação dos ciclos estrais.

\section{CONCLUSÕES}

A suplementação em creep feeding é uma alternativa capaz de aumentar o ganho médio diário e o peso à desmama de bezerros Nelore, aumentando também o peso de suas mães ao final da estação de monta, sem, no entanto, aumentar significativamente as taxas de gestação de primíparas quando estas iniciam a estação de monta com baixa condição corporal.

\section{REFERÊNCIAS BIBLIOGRÁFICAS}

BOGGS, D.L.; SMITH, R.R.; SCHALLES, B.E. et al. Effects of milk and forage intake on calf performance. J. Anim. Sci., v.51, p.550, 1980.

FORDYCE, J.; COOPER, N.J.; KENDALL, I.E. et al. Creep feeding and prepartum supplementation effects on growth and fertility of Brahman-cross cattle in the dry tropics. Aust. J. Exp. Agric., v.36, p.389-395, 1996.

JOLLY, P.D.; FITZPATRICK, L.A.; MAcDOUGALL, S. et al. Physiological effects of undernutrition on postpartum anoestrum in cows. In: 'Reproduction in Domestic Ruminants III'. J. Reprod. Fertil., v.49, suppl., p.477-492, 1994.

LEAL, T.C.; FREITAS, J.E. Correlação entre produção de leite e ganho de peso de bezerros da raça Charolesa. An. Téc. IPZFO, v.9, p.91-101, 1982.

LISHMAN, A.W.; LYLE, A.D.; SMITH; V.W. et al. Conception rate of beef cows and growth of suckling calves as influenced by date of calving and supplementary feeding. S. Afr. J. Anim. Sci., v.14, p.10-19, 1984a.

LISHMAN, A.W.; SNYMAN, J.W.; MOOLMAN, J.Z. Reconception and body-mass change of energy supplemented first-calver beef cows and growth of their creep-fed calves. S. Afr. J. Anim. Sci., v.14, p.20-15, 1984 b.

MARTIN, T.G.; LEMENAGER, R.P.; SRINIVASAN, G. et al. Creep feeding as a factor influencing performance of cow and calves. J. Anim. Sci., v.53, p.33-39, 1981.

NOGUEIRA, É.; MORAIS, M.G.; ANDRADE, V.J. et al. Valor nutritivo de pastagem de Brachiaria brizantha cv. Marandu sob pastejo contínuo durante o período de estação de monta, em propriedade do Mato Grosso do Sul. Ens. Ciênc., v.7, p.1015-1021, 2003a.

NOGUEIRA, É.; MORAIS, M.G.; ROCHA, E.D. et al. Produtividade e percentuais de caules e folhas de Brachiaria brizantha cv. Marandu sob pastejo contínuo durante o período de estação de monta em propriedade no Mato Grosso do Sul. Ens. Ciênc., v.7, p.841-846, 2003b. 
PACOLA, L.J.; NASCIMENTO, J.; MOREIRA, H.A. Alimentação suplementar de bezerros zebu: influência sobre a idade dos machos ao abate e das fêmeas à primeira cobrição. Bol. Ind. Anim., v.34, p.177-201, 1977.

PACOLA, L.J.; RAZOOK, A.G.; NETO, L.M.B. et al. Suplementação de bezerros em cocho privativo. Bol. Ind. Anim., v.46, n.2, p. 167-175, 1989.

RICHARDS, M.W.; WETTEMAN, R.P.; SCHOENEMANN, H.M. Nutritional anoestrus in beef cows: body weight change, body condition, luteinizing hormone in serum and ovarian activity. J. Anim. Sci., v.67, p.1520, 1989.

SIQUEIRA, J.G.; FONTES, C.A.A.F.; RIBEIRO, E.G. et al. Influência do Creep feeding sobre o desempenho de bezerros F1 Limousin-Nelore e F1 Belgian Blue-Nelore. In: REUNIÃO ANUAL DA SOCIEDADE BRASILEIRA DE ZOOTECNIA, 38., Piracicaba. Anais... Piracicaba: SBZ, 2001.
SPITZER, J.C.; MORRISON, D.G.; WETERMAN, R.P. Reproductive responses and calf birth and weaning weights as affected by body condition at parturition and postpartum weight gain in primiparous beef cows. J. Anim. Sci., v.73, p.1251-1257, 1995.

SWAYER, G.J.; BARKER, D.J.; MORRIS, R.J. Performance of young breeding cattle in commercial herds in the south-west of Western Australia 2. Liveweight, body condition, timing of conception and fertility in first-calf heifers. Aust. J. Exp. Agric., v.31, p.443-54, 1991.

USER'S guide: statistical analyses system. Release 6:11. Cary, NC: SAS Institute, 1996.

WILTBANK, J.N. Effect of nutrition and other factors on the reproduction heifers. In: ANNUAL BEEF CATTLE SHORT COURSE, 26., 1983, Gainesville. Proceedings... Gainesville: Institute of Food and Agricultural Sciences, 1983. p.63-68. 